\title{
Characteristics improvement of pressure transfer standard using a silicon resonant sensor
}

\author{
Hideaki Yamashita ${ }^{1}$, Hirokazu Nagashima ${ }^{1}$, Hideki Yamada ${ }^{1}$ \\ ${ }^{1}$ Yokogawa Test \& Measurement Corporation, 4-9-8 Myojin-cho, Hachioji-shi, Tokyo 192-8566, Japan
}

\section{ABSTRACT}

In the field of pressure measurement, numerous interlaboratory comparisons are carried out among National Metrology Institutes (NMIs) using a pressure transfer standard to verify the degrees of equivalence. Here, the Yokogawa electric corporation has been producing a series of digital manometers using a silicon resonant sensor developed independently. This sensor demonstrates excellent long-term stability and has thus been adopted as the pressure transfer standard by many NMIs and has been subsequently well received. The pressure transfer standard is known as the resonant silicon gauge (RSG) among NMIs. From December 2016, the National Metrology Institute of Japan (NMIJ), the Advanced Industrial Science and Technology (AIST) institute, and Yokogawa initiated a collaborative research with the aim of improving the characteristics of the RSGs and developing a portable transfer standard using a new silicon resonant sensor. The new RSG was adjusted using a standard device calibrated by either NMIJ or Yokogawa. The measurement values of the standard device were corrected with the calibration results and used as the standard values for adjustment of the new RSG. The linearity of the new RSG adjusted via the proposed method was improved compared with that of a conventional RSG.

Section: RESEARCH PAPER

Keywords: Transfer standard; international comparison; digital manometer; silicon resonant sensor; resonant silicon gauge

Citation: Hideaki Yamashita, Hirokazu Nagashima, Hideki Yamada, Characteristics improvement of pressure transfer standard using a silicon resonant sensor, Acta IMEKO, vol. 10, no. 1, article 38, March 2021, identifier: IMEKO-ACTA-10 (2021)-01-38

Editor: Momoko Kojima, NMIJ, Japan

Received May 15, 2020; In final form July 31, 2020; Published March 2021

Copyright: This is an open-access article distributed under the terms of the Creative Commons Attribution 3.0 License, which permits unrestricted use, distribution, and reproduction in any medium, provided the original author and source are credited.

Corresponding author: Hideaki Yamashita, e-mail: H.Yamashita@yokogawa.com

\section{INTRODUCTION}

The conclusion of the Mutual Recognition Arrangement of the Comité International des Poids et Mesures (CIPM-MRA) has promoted barrier-free trade in the globalised economy. The CIPM-MRA provides for the mutual recognition of the equivalence of national standards, with the equivalence ensured via international comparisons among the National Metrology Institutes (NMIs) [1], [2]. The international comparison is commonly carried out using a transfer standard, which is circulated to the NMIs in each country, with each NMI calibrating the transfer standard in accordance with their own national standards. The calibration results are then compared among the NMIs participating in the international comparison and the equivalence of the national standards is confirmed. In the international comparison of pressure measurement, a pressure balance is mainly used as the transfer standard [3]. However, a pressure balance is large, heavy, and extremely precise, meaning it is not easy to transfer the instrument as the transfer standard from one country to another. As such, the
NMIs, including the National Metrology Institute of Japan (NMIJ) and the Advanced Industrial Science and Technology (AIST) institute, took notice of a digital manometer that is smaller, lighter, more portable, and easier to handle than the pressure balance. Since the international comparison usually takes a year or longer, the transfer standard must demonstrate high stability. While the digital manometer provides less stability than the pressure balance, the NMIs have established a method to compensate for this shortfall. Here, the reliability of the transfer standard is ensured via redundancy using multiple digital manometers. The long-term stability of the digital manometer is compensated by correcting the calibration values using the results of long-term stability evaluations [3]. These methods have made it possible to use the digital manometer for the transfer standard, and a number of international comparisons using these transfer standards have already been conducted.

The digital manometers and pressure sensors developed by the Japanese electric company, Yokogawa, have been used for such transfer standards for international comparisons [4]-[6]. A pressure sensor is a digital manometer without a display and without operation keys. Yokogawa provides NMIs with various 
pressure sensors, including $1 \mathrm{kPa}$ differential pressure, $10 \mathrm{kPa}$ differential pressure and $100 \mathrm{kPa}$ absolute pressure models under the model name 265381/Z. Meanwhile, the 265381/Z is equipped with Yokogawa's silicon resonant sensor [7]-[9], with this type of manometer known as a resonant silicon gauge (RSG) among the NMIs [10]. Since the NMIs requested that the supply of the RSG be continued, the development of a new RSG has been initiated. The sensor structure of the new RSG is different from that of the conventional RSG, which requires the evaluation of basic performance (linearity, hysteresis, and repeatability). As such, Yokogawa initiated a collaborative research in conjunction with NMIJ/AIST, which involves national standards and precise evaluation techniques, to improve the basic performance and characteristics of the new RSG.

\section{SILICON RESONANT SENSOR}

Yokogawa's digital manometers are equipped with the silicon resonant sensor developed by the company itself [7]-[9], which is regarded as an RSG. This section provides an overview of this silicon resonant sensor.

The diaphragm, resonator and vacuum chamber covering the resonator, which constitute the silicon resonant sensor, are all formed from single crystal silicon using semiconductor processing technology. Two resonators are placed at positions where tension and compressive forces are generated on the diaphragm. The resonators are then excited by a magnetic circuit, while since they are located in the vacuum chamber, any vibration attenuation is suppressed, and a high $\mathrm{Q}$ factor can be obtained. When the silicon diaphragm is deformed by the application of pressure, the resonators are distorted and their resonant frequencies change [7]. The frequencies of the two resonators are then detected and the difference is converted into a pressure value. In addition, the RSG output value is corrected using correction factors calculated from the relationship between the applied pressure and the frequencies of the resonators. The silicon resonant sensor has excellent reproducibility and exhibits only slight secular change since its pressure detection part is formed of single crystal silicon [7], which is an elastic material. This characteristic contributes to the achievement of high stability.

Figure 1 shows the calibration results of a $10-\mathrm{kPa}$ differential pressure RSG, which was equipped with Yokogawa's silicon resonant sensor (10-kPa RSG), in relation to the company's double pressure balances. The range of calibration values over a two-year period was within $0.06 \mathrm{~Pa}$, while the long-term stability required for a transfer standard demonstrated good results. However, inflection points were observed on the calibration

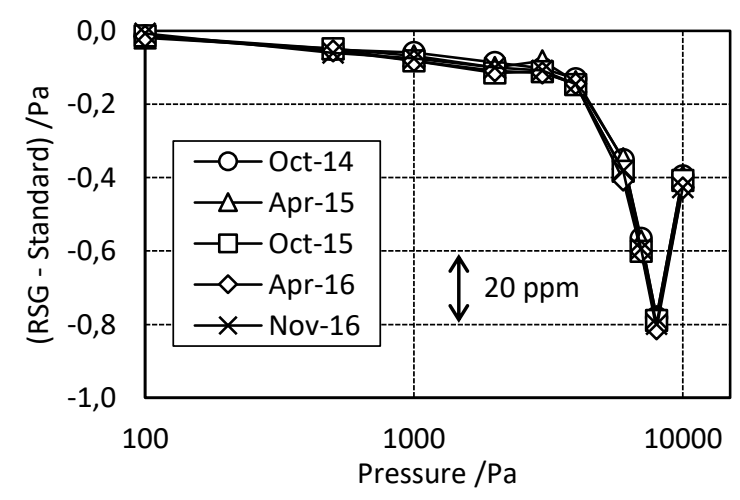

Figure 1. Calibration results for the 10-kPa RSG \#A from 2014 to 2016. curves [11]. The Yokogawa 10-kPa RSG, which has been used as a transfer standard, has the same tendency. The stability and hysteresis, which are crucial elements of the basic performance, are determined by the structure of the sensor in terms of, for example, the detection part of the silicon resonant sensor and the diaphragm of the pressure detector section. Meanwhile, the linearity can be improved using the adjustment method. With all this in mind, we developed a new RSG and worked on the confirmation of the basic performance and the improvement in linearity via adjustment.

\section{COLLABORATIVE RESEARCH WITH NMIJ/AIST}

The aim is to develop an RSG that can be used for a transfer standard for international comparison, one that can be continuously supplied. In order to confirm the basic performance of the RSG, development using national standards is necessary. In 2016, Yokogawa initiated a collaborative research alongside with the NMIJ/AIST, which has enabled an evaluation based on national standards.

An RSG corresponding to national standards or an RSG directly calibrated by these standards was used as the standard device for adjustment. The RSG measured value was corrected via a calibration value and was subsequently used as a standard value. Repeated simulations were then conducted using the standard value and the frequency data of the resonators and attempted to optimise the pressure points used for the adjustment. The effect of the adjustment was confirmed by directly comparing the adjusted RSG with the national standards. The development was carried out according to the following procedure:

1) Yokogawa produce an RSG (referred to as RSG \#A).

2) Calibrate the RSG \#A (the $10 \mathrm{kPa}$ by Yokogawa, the others by NMIJ/AIST).

3) Yokogawa feed back the result of 2), correct the output of RSG \#A and use it as the standard device to newly adjust another RSG (referred to as RSG \#B).

4) $\mathrm{NMIJ} / \mathrm{AIST}$ evaluate the RSG \#B adjusted in 3) to confirm the effect of the adjustment.

Meanwhile, the following outlines Yokogawa's procedure for adjusting the RSGs:

1) Place the RSG to be adjusted in a temperature chamber and the standard device outside the temperature chamber.

2) Apply pressure from a pressure controller to the RSG and the standard device.

3) Obtain the frequencies of the resonators of the RSG and the pressure measurements of the standard device by changing the temperature and pressure.

4) The coefficients of the approximation formula calculated from the acquired data are written to the internal memory of the RSG as a correction factor.

In this research, multiple RSGs were adjusted at the same time. This is the same method as that used in the manufacturing process for Yokogawa's digital manometers. Being able to use the existing method to adjust RSGs without requiring a special procedure and without the need for a large amount of time or expenditure is of great benefit to the continuous provision of RSGs.

Figure 2 shows the developed prototype of the 10-kPa RSG. While it has input ports on the front, it features no display or operation keys. The acquisition of the pressure values and of various settings is performed through USB communication with 


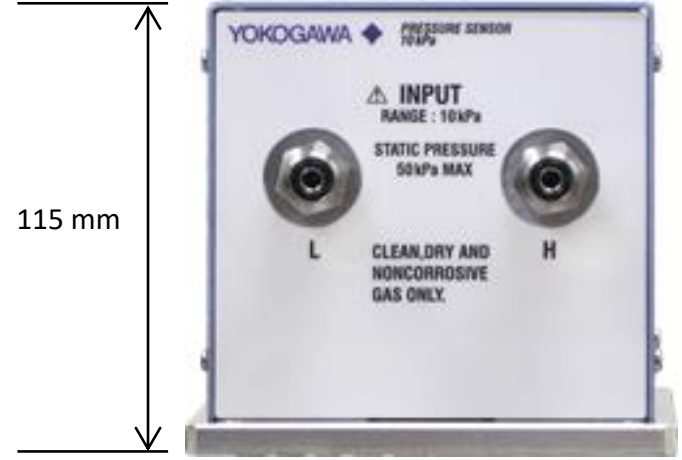

Figure 2. Photograph of the prototype 10-kPa RSG.

a PC, with a USB port provided on the rear. The external appearance is the same regardless of the pressure range.

In this collaborative research, we developed RSGs characterised by a $10 \mathrm{kPa}$ differential pressure, $200 \mathrm{kPa}$ gauge pressure, $1,000 \mathrm{kPa}$ gauge pressure, $3,500 \mathrm{kPa}$ gauge pressure and $130 \mathrm{kPa}$ absolute pressure. The NMIJ/AIST evaluated certain characteristics of the RSGs, including the linearity and stability. The following sections introduce the characteristics of the RSGs as obtained via the evaluations.

\section{RESONANT SILICON GAUGE CHARACTERISTICS}

\subsection{Calibration results and linearity of the 10-kPa RSG}

The 10-kPa RSG \#B was adjusted using the 10-kPa RSG \#A shown in Figure 1 as the standard device. For the evaluation, calibration was performed via direct comparison with the generated differential pressure using double pressure balances. Ten points of measurement pressure were selected in the range of $1 \mathrm{~Pa}-10 \mathrm{kPa}$, while the line pressure was $100 \mathrm{kPa}$. The 'ABABA' method [12], [13] was used for the differential pressure generation sequencing, with the measurement performed during the pressure ascending processes. The pressure was ascended five times and measurement was carried out once for each. The average of the measured values was then used as the calibration pressure value.

Figure 3 shows the calibration results of two units of RSG \#B. The calibration data shown in the figures hereafter - with the exception of those shown in Figure 8 - were provided by NMIJ/AIST. The two units were calibrated at the same time and the maximum deviation in the NMIJ/AIST pressure standards was $0.07 \mathrm{~Pa}$. The linearity of the RSG \#B was greatly improved in comparison to the RSG \#A, with the maximum difference

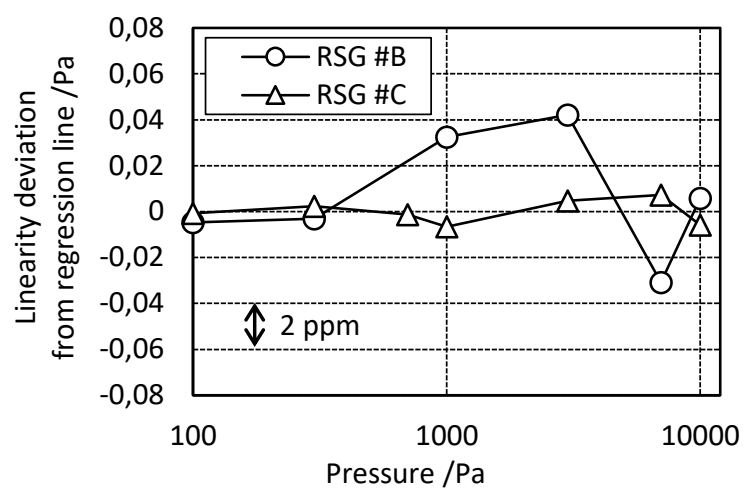

Figure 4. Linearity deviations from a regression line of the $10-\mathrm{kPa}$ RSGs \#B and \#C. The RSG \#C was adjusted using the RSG \#B as the standard.

between the two units $0.03 \mathrm{~Pa}$. It was confirmed that this difference could be obtained by simultaneously adjusting multiple RSGs in combination with the pressure controller and standard device. In fact, it is possible to supply RSGs with small variations in characteristics when multiple RSGs are required to improve the reliability of the transfer standard.

The RSG \#B was adjusted using the RSG \#A calibrated by Yokogawa as the standard device. While the RSG \#A correlated with the national standards, it lay deep in the attendant hierarchy. Therefore, the RSG \#C was adjusted using the RSG \#B calibrated in terms of the national standards as the standard device. The calibration results of the RSGs \#B and \#C obtained using the NMI/AIST pressure standards were then compared with the regression lines, and linearity comparisons were made, with the results shown in Figure 4. The RSG \#C exhibited further improved linearity compared to the RSG \#B.

\subsection{Short- and long-term stability of the 10-kPa RSG}

Figure 5 shows each of the measurement results for the five pressure ascending processes, as described in subsection 4.1. which confirmed the short-term stability of the $10-\mathrm{kPa}$ RSG during the calibration process in terms of the repeatability. Even in the measurement results at $10 \mathrm{kPa}$, which had the largest variation, the standard deviation was only $0.028 \mathrm{~Pa}$, which was 3 ppm or less with respect to the full scale.

Figure 6 shows the results of two calibrations performed 10 months apart to confirm the long-term stability, with the calibration results aligning within $0.04 \mathrm{~Pa}$. It was confirmed that the stability was excellent, both in the short-term and in the longterm, through the evaluation performed by NMIJ/AIST, where the procedure was highly controlled.

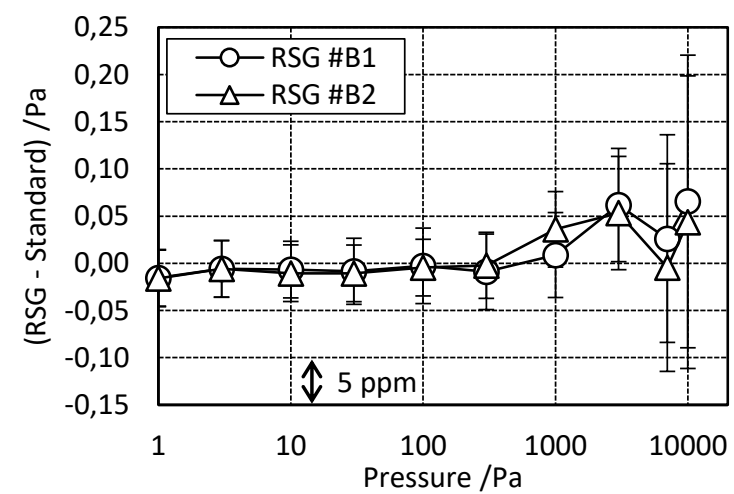

Figure 3. Calibration results for the 10-kPa RSGs \#B1 and \#B2. The error bars refer to expanded $(k=2)$ uncertainties.

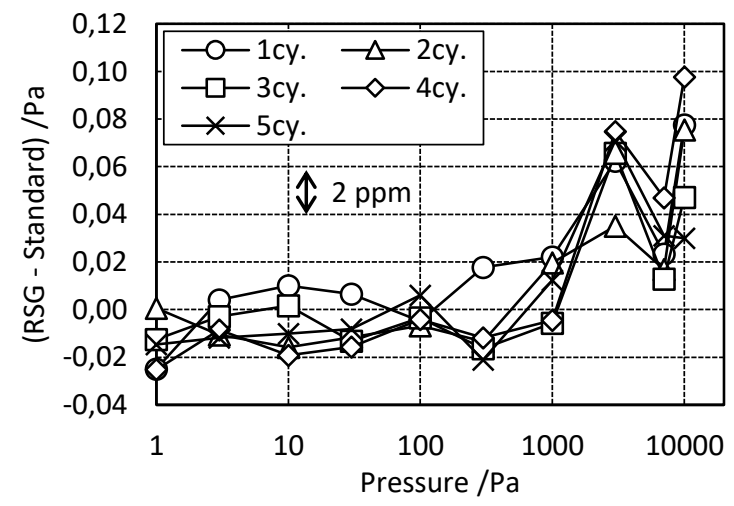

Figure 5. Short-term stability of the 10-kPa RSG \#B. 


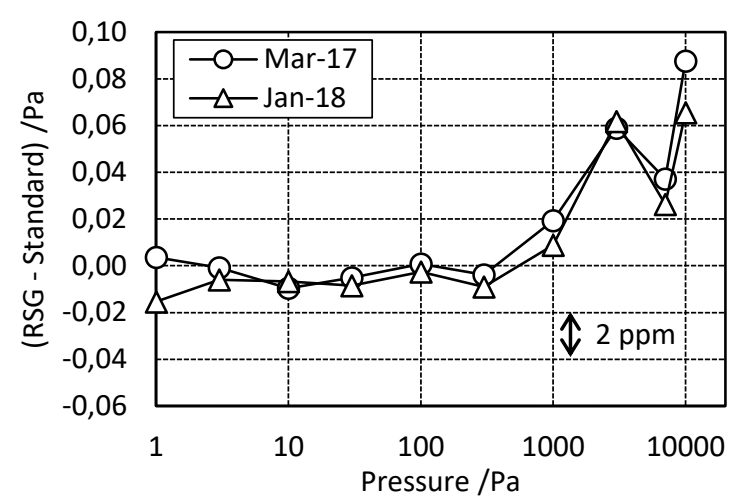

Figure 6. Long-term stability of the 10-kPa RSG \#B.

\subsection{Influence of line pressure and temperature of the 10-kPa RSG}

While the line pressure of a differential pressure gauge at the time of calibration is usually $100 \mathrm{kPa}$, the gauge may be used with the line pressure set to vacuum in the international comparison of absolute pressure ranges [5], [10]. In this case, as the line pressure differed from that at the time of calibration by $100 \mathrm{kPa}$, it was necessary to check the influence of any changes in line pressure in advance [11], [13]. The RSG \#B was calibrated with line pressures of 75, 100 and $125 \mathrm{kPa}$, and the influence per line pressure of $100 \mathrm{kPa}$ was estimated from the calibration results.

Figure 7 shows the change in calibration results when the line pressure was altered by $100 \mathrm{kPa}$. A change in the span was observed, with the amount of change in the calibration results $0.6 \mathrm{~Pa}$ at $10 \mathrm{kPa}$. The RSG \#B was adjusted such that the zero point was not affected by the line pressure, while the span was not adjusted. There is the possibility that the line pressure dependence of the span can be corrected by changing the line pressure and acquiring specific span data, and then including it in the adjustment parameters. Furthermore, since the characteristics are linear, it may also be possible to make corrections using regression lines. These approaches should be examined in future research.

There exists a transfer standard using a temperaturecontrolled enclosure to reduce the influence of the ambient temperature [5], [10]. If the temperature characteristics of an RSG that is incorporated in the transfer standard are improved, the influence of the temperature can be minimised with the use of enclosure, while there is also the possibility that the enclosure can be omitted. The correction factor of the RSG \#B was calculated using its adjustment parameters that were obtained in the range of $-10^{\circ} \mathrm{C}-50{ }^{\circ} \mathrm{C}$.

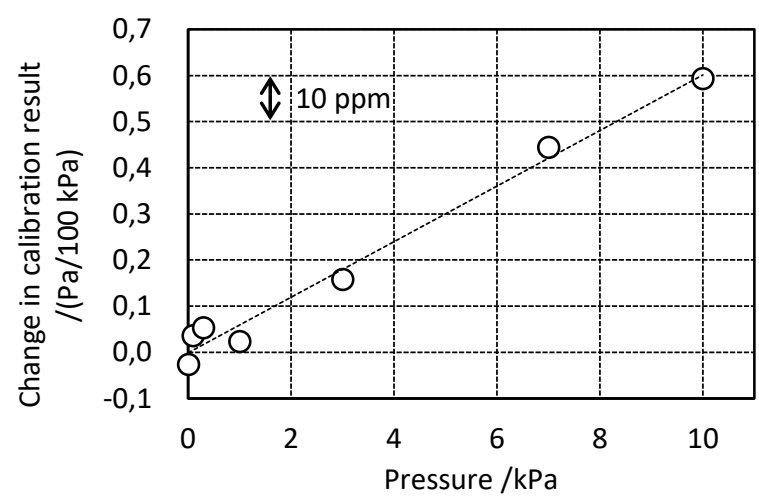

Figure 7. Influence of the line pressure on the calibration results for the 10$\mathrm{kPa}$ RSG \#B. Change in calibration results per $100 \mathrm{kPa}$ of line pressure.

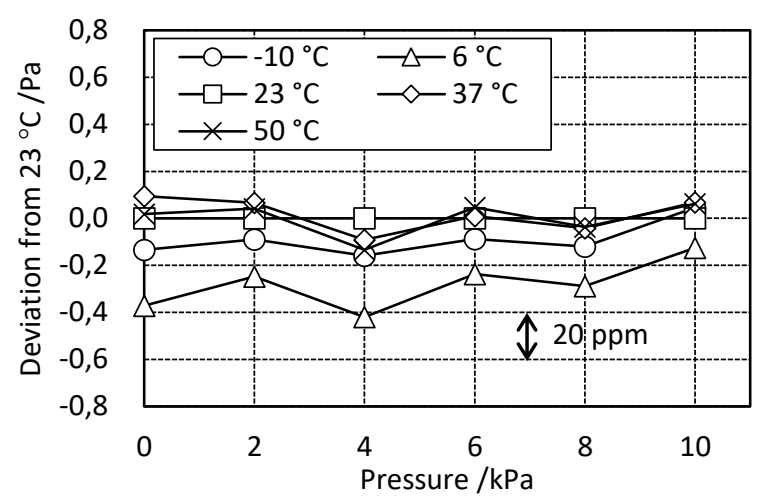

Figure 8. Influence of temperature variation on output of the 10-kPa RSG \#B.

Figure 8 shows the results of the measurement of the inputoutput characteristics at certain temperatures carried out by Yokogawa, with deviations from $23{ }^{\circ} \mathrm{C}$. In the range of $-10^{\circ} \mathrm{C}-$ $50{ }^{\circ} \mathrm{C}$, the maximum temperature coefficient was $0.03 \mathrm{~Pa} /{ }^{\circ} \mathrm{C}$. However, while the adjustment range was set to $-10{ }^{\circ} \mathrm{C}-50{ }^{\circ} \mathrm{C}$ in this research, there is no such exact temperature range in the international comparison environment [5]. It is believed to be possible to reduce the influence of the temperature by optimising the adjustment temperature range.

\subsection{Characteristics of other pressure range RSGs}

The international comparison of pressure measurement is performed over a wide pressure range [3], [14]. This section reports the characteristics of the $130-\mathrm{kPa}$ absolute pressure RSG (130-kPa abs RSG) and the $3,500-\mathrm{kPa}$ gauge pressure RSG

(a)

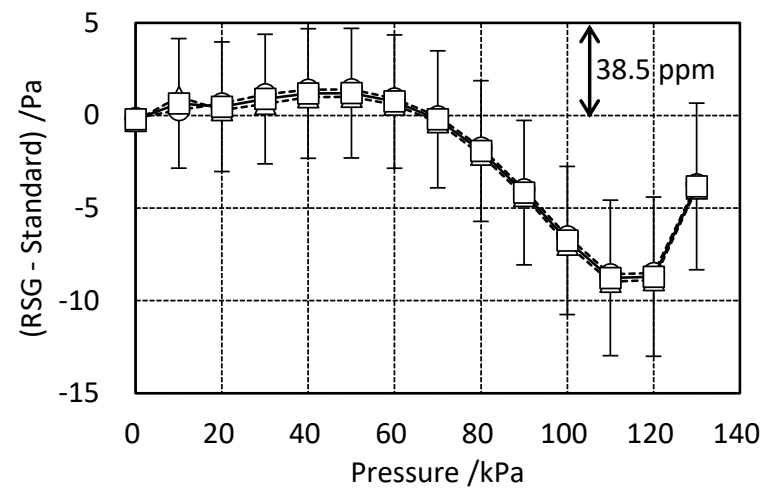

(b)

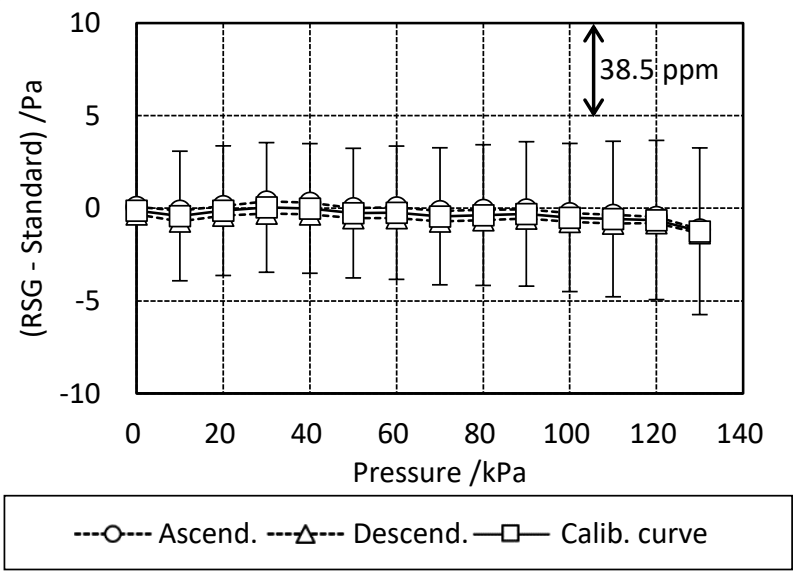

Figure 9. Calibration results for the 130-kPa abs RSG (a) \#A and (b) \#B. The error bars refer to expanded $(k=2)$ uncertainties. 


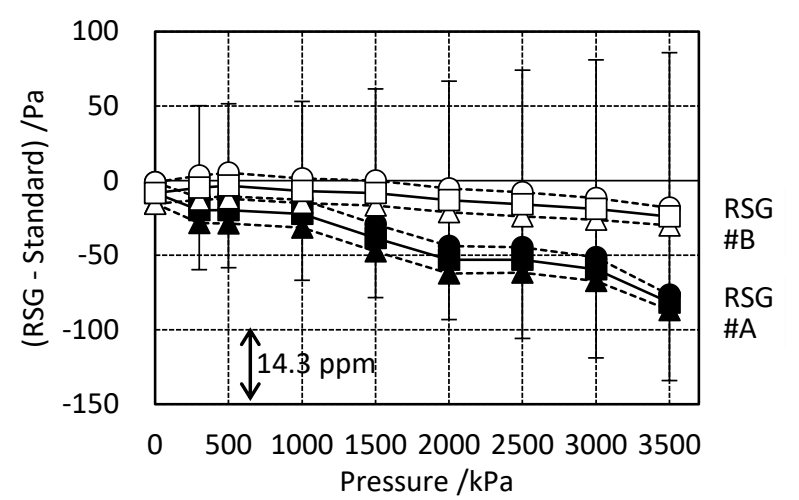

Ascend.

Figure 10. Calibration results for the 3,500-kPa RSGs \#A and \#B. The error bars refer to expanded $(k=2)$ uncertainties.

(3,500-kPa RSG), which were developed in view of the expansion of RSGs into ranges other than $10 \mathrm{kPa}$. An adjustment was made using a pressure controller and a standard device following the procedure used for the $10-\mathrm{kPa}$ RSG, with the RSG \#A calibrated by NMIJ/AIST used as the standard device. For the evaluation of the adjusted RSG \#B, calibration was performed by direct comparison with the NMIJ/AIST pressure balance. The measurements were carried out in each of three calibration cycles with the pressure both ascending and descending. The average of the measured values was then used as the calibration pressure value.

(a)

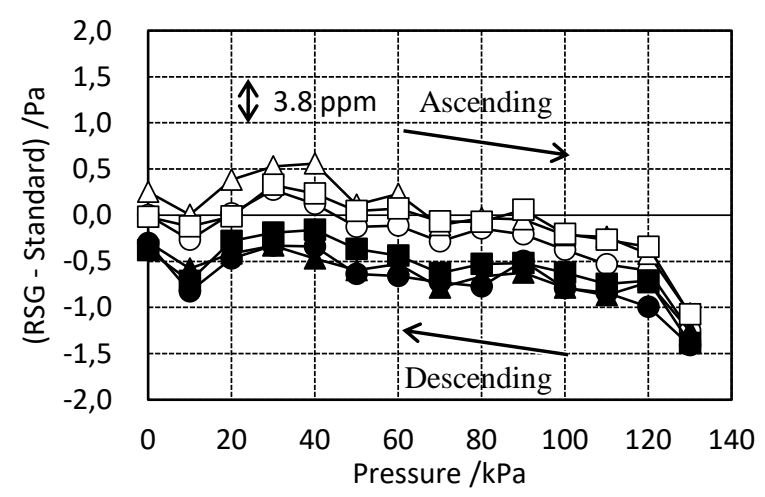

(b)
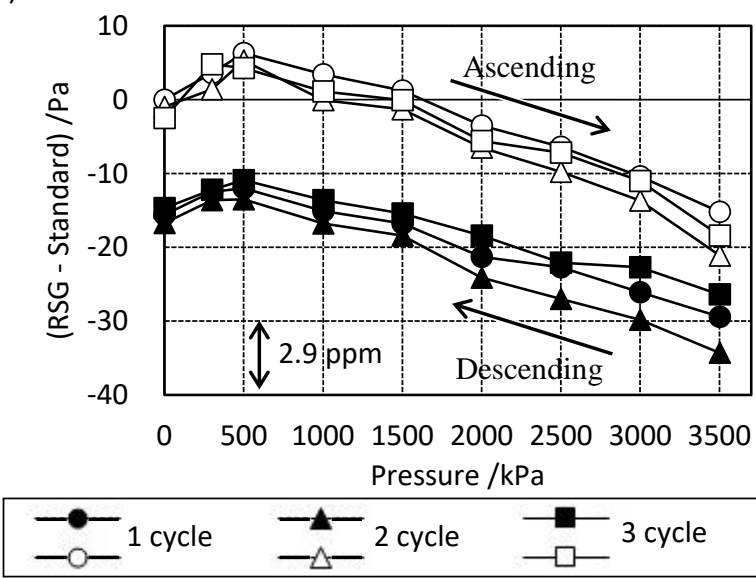

Figure 11. Short-term stability of (a) the $130-\mathrm{kPa}$ abs RSG \#B and (b) the 3,500-kPa RSG \#B.
Figure 9 and Figure 10 show the calibration results for the standard device RSG \#A and the adjusted device RSG \#B. Here, the deviation from the national standards could be maintained at within $10 \mathrm{ppm}$ of the full scale. Specifically, a large effect was observed in improving the linearity of the $130-\mathrm{kPa}$ abs RSG. This indicates that the method using a standard device calibrated by the national standards for adjustment is effective for RSGs of various pressure ranges.

Figure 11 shows the input-output characteristics in three cycles for the 130-kPa abs RSG \#B and the 3,500-kPa RSG \#B. Here, the short-term stability was confirmed. The standard deviations of the three measurements both the \#B RSGs were $0.5 \mathrm{~Pa}$ and $4.0 \mathrm{~Pa}$, respectively, and the relative value of standard deviation with respect to the full scale was equivalent to that of the $10-\mathrm{kPa}$ RSG. It was also confirmed that the reproducibility of repeated measurements was excellent.

The adjustment method also proved to be effective in all the RSGs, including the $10-\mathrm{kPa}$ RSG. Therefore, the method was applied to the production of standard devices for the new digital manometer, which was labelled MT300 and features an array of ranges [15].

\section{CONCLUSIONS}

Through the collaborative research carried out in conjunction with NMIJ/AIST, we attempted to improve the characteristics of the RSGs used as the transfer standards for international comparison. The developed RSGs were evaluated by NMIJ/AIST through direct comparison with the national standards. The method through which the standard device was corrected via the calibration value and used for adjustment purposes was adopted to improve the linearity.

As described in subsection 4.1., for the 10-kPa RSG, the linearity with respect to the national standards was greatly improved and adjustments with a slight difference between the two units were possible. Meanwhile, in subsection 4.2, the unit's excellent stability was confirmed in both the short-term and long-term. The performance of the transfer standard is expected to be improved by replacing the conventional RSG with the new RSG. Furthermore, the line pressure and the temperature dependence described in subsection 4.3 can be expected to be improved by optimising the adjustment conditions, an approach that should be examined and evaluated in future research.

As described in subsection 4.4., the adjustment method proved to be effective through the evaluation of the $10-\mathrm{kPa}$ RSG as applied to the RSGs of other ranges. A deviation of within 10 ppm of the full scale pertaining to the national standards was achieved through making adjustments using the standard device directly calibrated in terms of the national standards. We believe it is possible to use RSGs in international comparisons covering a wide pressure range.

The transfer standard using a digital manometer has improved the efficiency of international comparisons related to pressure measurement. The use of digital manometers in the area of international comparisons is expected to increase in the future. As such, digital manometers that are compact and lightweight and which have excellent characteristics will be indispensable, and Yokogawa has the accumulated RSG technology to achieve this. We will continue to contribute to the advancement of pressure measurement in both Japan and overseas through developments that include the characteristics improvement and range expansion of RSGs. 


\section{ACKNOWLEDGEMENTS}

The authors would like to thank the members of the NMIJ/AIST Pressure and Vacuum Standards Group for arranging the evaluation, compiling the results, and providing helpful advice in the collaborative research.

\section{REFERENCES}

[1] National Institute of Advanced Industrial Science and Technology, Center for Quality Management of Metrology, International Cooperation Office website. Online [Accessed 13 May 2020]

https://unit.aist.go.jp/qualmanmet/nmijico/en/comp/

[2] Ministry of Economy, Trade and Industry. Online (in Japanese) [Accessed 13 May 2020]

https://www.meti.go.jp/shingikai/keiryogyoseishin/pdf/g50913 a46j.pdf

[3] T. Kobata, M. Kojima, H. Kajikawa, Improvement of reliability in pressure measurements and international mutual recognition Incorporation of industrial digital pressure gauges to the national metrology system, Synthesiology - English edition 4 (2012), pp. 212-226. Online [Accessed 29 March 2021]

https://www.aist.go.jp/pdf/aist e/synthesiology e/vol4 no4/v ol04 04 p212 p226.pdf

[4] T. Kobata, M. Kojima, K. Saitou, M. Fitzgerald, D. Jack, C. Sutton, Final report on key comparison APMP.M.P-K5 in differential pressure from $1 \mathrm{~Pa}$ to $5000 \mathrm{~Pa}$, Metrologia 44 Tech. Suppl. 07001 (2007), 52 pp. Online [Accessed 23 March 2021] iopscience.iop.org/article/10.1088/0026-1394/44/1A/07001

[5] J. Ricker, J. Hendricks, T. Bock, P. Dominik, T. Kobata, J. Torres, I. Sadkovskaya, Final report on the key comparison CCM.PK4.2012 in absolute pressure from $1 \mathrm{~Pa}$ to $10 \mathrm{kPa}$, Metrologia 54 Tech. Suppl. 07002 (2017), 37 pp. Online [Accessed 23 March 2021]

iopscience.iop.org/article/10.1088/0026-1394/54/1A/07002

[6] J. Hendricks, J. Ricker, D. Olson, Protocol CCM - International key comparison in absolute pressure (1 $\mathrm{Pa}$ to $10 \mathrm{kPa}$ ) CCM.P K4.2012, The BIPM key comparison database. Online [Accessed 13 May 2020]

https://www.bipm.org/kcdb/comparison/doc/download/1194 /ccm.p-k4.2012 technical protocol.pdf
[7] K. Ikeda, H. Kuwayama, T. Kobayashi, T. Watanabe, T. Yoshida, T. Nishikawa, K. Harada, Silicon pressure sensor integrates resonant strain gauge on diaphragm, Sensors and Actuators A: Physical 21 (1990), pp. 146-150. DOI: $10.1016 / 0924-4247(90) 85028-3$

[8] T. Saigusa, S. Gotoh, H. Kuwayama, M. Yamagata, DPharp series electronic differential pressure transmitters, Yokogawa Technical Report English Edition 15 (1992), pp. 30-37.

[9] T. Ishikawa, T. Odohira, M. Nikkuni, E. Koyama, T. Tsumagari, R. Asada, New DPharp EJX series pressure and differential pressure transmitters, Yokogawa Technical Report English Edition 37 (2004), pp. 9-14. Online [Accessed 29 March 2021] https://www.yokogawa.com/about/researchdevelopment/rd te report/rd tr report a00037/

[10] J. Hendricks, A. Miiller, Development of a new high-stability transfer standard based on resonant silicon gauges for the range $100 \mathrm{~Pa}$ to $130 \mathrm{kPa}$, Metrologia 44 (2007), pp. 171-176. DOI: $10.1088 / 0026-1394 / 44 / 3 / 002$

[11] M. Kojima, T. Kobata, Characterisation of differential pressure gauges focusing on calibration curve and line pressure dependency, Proc. of the 29th Sensing Forum (2012), pp. 9-13. (in Japanese).

[12] M. Kojima, T. Kobata, K. Saitou, M. Hirata, Development of small differential pressure standard using double pressure balances, Metrologia 42 (2005), pp. 227-230. DOI: $10.1088 / 0026-1394 / 42 / 6 /$ S18

[13] M. Kojima, K. Saitou, T. Kobata, Study on calibration procedure for differential pressure transducers, Proc. of the IMEKO $20^{\text {th }}$ TC3, 3 ${ }^{\text {rd }}$ TC16 and $1^{\text {st }}$ TC22 International Conference Cultivating metrological knowledge, Merida, Mexico, 27 - 30 November 2007. Online [Accessed 13 May 2020] https://www.imeko.org/publications/tc16-2007/IMEKOTC16-2007-044u.pdf

[14] M. Kojima, T. Kobata, Calibration system and traceability of low pressure and vacuum standards in Japan, Journal of the Vacuum Society of Japan 59 (2016), pp. 352-359. (in Japanese).

[15] Yokogawa Test \& Measurement Corporation, Brochure for the MT300 digital manometer, 2020. Online [Accessed 23 March 2021]

https://tmi.yokogawa.com/solutions/products/other-testmeasurement-instruments/manometers/mt300-digitalmanometer/ 